\title{
Dual anti-HSV and anti-HIV activity of the lantibiotic Labyrinthopeptin A1
}

\author{
Geoffrey Férir ${ }^{1 *}$, Mariya I Petrova ${ }^{2}$, Graciela Andrei ${ }^{1}$, Robert Snoeck ${ }^{1}$, Mark Brönstrup ${ }^{3}$, Roderich D Süssmuth", \\ Dominique Schols ${ }^{1}$
}

From International Symposium HIV and Emerging Infectious Diseases 2014

Marseille, France. 21-23 May 2013

\section{Background}

It has been shown that genital lesions and altered innate mucosal immunity caused by HSV-2 are important cofactors to increase the rate of HIV transmission and infection. Therefore, a product that inhibits HIV and HSV would have potential benefits in the prophylaxis against these sexually transmitted viruses. The labyrinthopeptin A1 (LabyA1) is a prototype peptide of a novel class of carbacyclic lantibiotics. Here, we extensively evaluated LabyA1 for its broad-spectrum activity against HIV and HSV.

\section{Methods}

Replication of HIV-1, HIV-2 and drug (e.g. tenofovir, maraviroc, raltegravir, saquinavir)-resistant viruses were evaluated in CD4+ T cell lines and in PBMCs. LabyA1 was also tested against HSV-1 and HSV-2 and HSV-resistant viruses (such as acyclovir). It was tested also in combination with other classes of anti-HIV/HSV drugs. EC50 values and potential synergy levels were calculated using CalcuSyn software. Potential cellular side-effects, cytokine induction, toxicity and growth inhibitions were also investigated.

\section{Results}

LabyA1 exhibited a consistent and broad anti-HIV activity (EC50: 0.70-3.3 $\mu \mathrm{M}$ ) and anti-HSV activity (EC50: 0.29-2.8 $\mu \mathrm{M})$. LabyA1 also inhibited viral cell-cell transmission between persistently HIV-infected T cells and uninfected CD4+ T cells (EC50: $2.5 \mu \mathrm{M}$ ) and inhibited the transmission of HIV captured on DC-SIGN to CD4+ T cells (EC50: $4.1 \mu \mathrm{M})$. LabyA1 behaves as a novel type of viral entry inhibitor. LabyA1 also demonstrated additive to synergistic effects in its anti-HIV-1 and anti-HSV-2 activity with anti(retro)viral drugs in dual combinations such as tenofovir, acyclovir, saquinavir, raltegravir and enfuvirtide. LabyA 1 was equally active against all drug-resistant HIV and HSV strains. It did not induce any inflammatory cytokines/chemokines and did not affect the growth of vaginal Lactobacilli.

\section{Conclusions}

LabyA1 has profound dual antiviral activity. Based on the lack of toxicity on the vaginal Lactobacillus strains and its synergistic/additive profile in combination with all approved anti(retro)virals, it deserves further attention as a potential microbicide candidate in the prevention of sexually transmitted (HIV/HSV) diseases.

\section{Authors' details \\ ${ }^{1}$ Rega Institute for Medical Research, University of Leuven, Leuven, Belgium. ${ }^{2}$ Center of Microbial and Plant Genetics, University of Leuven, Leuven, \\ Belgium. ${ }^{3}$ Helmholtz Center for Infection Research, Braunschweig, Germany. ${ }^{4}$ Technische Universität Berlin, Fakultät II - Institut für Chemie, Berlin, Germany.}

Published: 23 May 2014

\section{doi:10.1186/1471-2334-14-S2-P79}

Cite this article as: Férir et al:: Dual anti-HSV and anti-HIV activity of the lantibiotic Labyrinthopeptin A1. BMC Infectious Diseases 2014 14(Suppl 2): P79.

${ }^{1}$ Rega Institute for Medical Research, University of Leuven, Leuven, Belgium Full list of author information is available at the end of the article 